\title{
A generalized acoustic analogy
}

\author{
M. E. Goldstein \\ National Aeronautics and Space Administration \\ Glenn Research Center \\ 21000 Brookpark Road \\ Cleveland, Ohio, United States 44135
}

\begin{abstract}
The purpose of this article is to show that the Navier-Stokes equations can be rewritten as a set of linearized inhomogeneous Euler equations (in convective form) with source terms that are exactly the same as those that would result from externally imposed shear stress and energy flux perturbations. These results are used to develop a mathematical basis for some existing and potential new jet noise models by appropriately choosing the 'base flow' about which the linearization is carried out.
\end{abstract}

\section{Introduction}

Lighthill $(1952,1954)$ provided a systematic basis for predicting jet noise when he rearranged the NavierStokes equations into the form of a linear wave equation for a medium at rest with a quadrupole-type source term, which includes a pressure/density contribution that Lilley (1974) showed to be more appropriately described by a dipole-type source. The crucial step in this so-called acoustic analogy approach amounts to assuming that the source term is in some sense known or that it can at least be modeled in an approximate fashion. While Lighthill's strategy turned out to be remarkably successful in predicting the gross features of the sound radiation from turbulent air jets, the engine manufacturers soon realized that they needed a much more sensitive tool with the capability of predicting how even relatively small changes in the flow would affect the radiated sound. This resulted in a number of attempts to improve the Lighthill approach. Early efforts were focused on accounting for mean flow interaction effects. Phillips (1960), Lilley (1974), and many others, sought to accomplish this by rearranging the Navier-Stokes equations into the form of an inhomogeneous convective or moving medium wave equation rather than the inhomogeneous stationary medium wave equation originally proposed by Lighthill.

But, jet noise reduction technology is still largely empirical and the most advanced industrial noise prediction methods, such as GE's MGB approach (Balsa et al. 1978), which is based on Lilley's extended acoustic analogy, 
may do a reasonable job of predicting the noise from simple round jets, but appear to be incapable of predicting the changes in the sound field that occur when noise suppression devices are deployed and therefore cannot be used to accomplish their intended purpose - the evaluation of the acoustic performance of these devices. Tam and Auriault (1999) recently developed a semi-empirical model that has successfully predicted the sound at angles near 90 degrees in a number of cases, but it does not predict the peak noise and has not as yet been used as a design tool. It is therefore still necessary to develop noise prediction methods that exhibit the required sensitivity to relatively small flow changes.

The most obvious approach would be to use large-scale numerical simulation, i.e., DNS. However, as has been frequently pointed out, the number of mesh points needed to fully resolve any turbulent flow is proportional to the Reynolds number $R$ based on the characteristic length scale of the turbulent eddies raised to the nine-fourths power. But since $R$ is of the order of $10^{5}$ to $10^{7}$ for typical jet engine exhaust flows, this means that $10^{12}$ to $10^{15}$ grid points would be needed to resolve all of the relevant length scales. This is beyond the capabilities of currently available machines and the community has been forced to acknowledge that meaningful predictions will have to be based on some sort of combined analytical-modeling method (such as the extended acoustic analogy approaches referred to above) or some approximate numerical method such as LES or perhaps some combination of the two. A number of such approaches have recently appeared in the literature.

The present paper is an attempt to provide a rational basis for these approaches. It begins by showing that the Navier-Stokes equations can always be recast into the convective form of the linearized Navier-Stokes (LNS) equations but with modified dependent variables, with the viscous stress perturbation replaced by a certain generalized Reynolds stress and, with the heat flux perturbation replaced by a generalized stagnation enthalpy flux. The 'base flow' about which the equations are linearized can be any solution to a very general class of inhomogeneous Navier-Stokes equations with arbitrarily specified source strengths. These exact results are then used, along with suitable choices for the base flow source strengths, to show how many of the recent and some of the more frequently used classical approaches to the jet noise problem can be put on a more rational basis and, more importantly, extended in new directions. The LNS equations are, of course, still nonlinear but the nonlinearity is effectively contained in the generalized Reynolds stresses and enthalpy flux-which also contain contributions from the base flow sources. The focus is on obtaining useful exact results and discussion of modeling and/or numerical issues (and the obligatory experimental comparisons) is only included when it relates to base flow selection. 
The acoustic analogy-type approaches and their extensions roughly correspond to treating the generalized stresses and enthalpy flux as known source terms that can be estimated or modeled as in the original Lighthill analysis. However, there are also cases where the base flow sources, which can be determined as part of the base flow computation, provide the dominant contribution to these quantities. This is most significant when the base flow is non-radiating (but, of course, unsteady) because the residual flow can then be identified with the acoustic part of the motion. The most obvious way of choosing the (unsteady) non-radiating base flow is to require that it be incompressible — but there are better choices, which are described in $\S 3.4$ below. This leads to a different approach to jet nose modeling (related to the choice of base flow), which has not, as yet, been exploited. There are also cases where a radiating base flow is the most appropriate choice. These are described in $\S 3.3$.

The fundamental equations are derived in $\S 2$ and used to justify and generalize various noise prediction methods in § 3.A version of the classical parallel mean flow approach (i.e. the Lilley's equation approach) is recovered in section 3.1 and certain conceptual difficulties are pointed out. Section 3.2 shows how these can be overcome by introducing non-parallel mean flow affects. The resulting modifications can significantly change the predictions of the theory.

Some of the material in this paper was presented in preliminary form in Goldstein (1999, 2000, 2002). But the present work is based on a more intuitively appealing form of the fundamental equations and a number of new results are derived.

\section{The LNS equations}

To obtain the fundamental equations, we begin with the Navier-Stokes equations in conservation law form:

$$
\begin{gathered}
\frac{\partial \rho}{\partial t}+\frac{\partial}{\partial x_{j}} \rho v_{j}=0 \\
\frac{\partial}{\partial t} \rho v_{i}+\frac{\partial}{\partial x_{j}} \rho v_{i} v_{j}+\frac{\partial p}{\partial x_{i}}=\frac{\partial}{\partial x_{j}} \sigma_{i j} \\
\frac{\partial}{\partial t}\left(\rho h_{o}-p\right)+\frac{\partial}{\partial x_{j}} \rho v_{j} h_{o}=-\frac{\partial q_{j}}{\partial x_{j}}+\frac{\partial}{\partial x_{j}} v_{i} \sigma_{i j}
\end{gathered}
$$

where 


$$
h_{o} \equiv h+\frac{1}{2} v^{2}
$$

denotes the stagnation enthalpy, $h$ denotes the enthalpy, $t$ denotes the time, $\boldsymbol{x} \equiv\left\{x_{1}, x_{2}, x_{3}\right\}$ are Cartesian coordinates, $p$ denotes the pressure, $\rho$ denotes the density, $v=\left\{v_{1}, v_{2}, v_{3}\right\}$ is the fluid velocity, $\sigma_{i j}$ is the viscous stress tensor, and $q_{i}$ is the heat flux vector. The dependent variables are required to satisfy an equation of state which, for definiteness, is taken to be the ideal gas law:

$$
p=\rho R T, \quad h=c_{p} T
$$

where $R=c_{p}-c_{v}$ is the gas constant, $c_{p}$ and $c_{v}$ are the specific heats at constant pressure and volume and $T$ is the absolute temperature.

The dependent variables

$$
\rho=\bar{\rho}+\rho^{\prime}, \quad p=\bar{p}+p^{\prime}, \quad h=\widetilde{h}+h^{\prime}, \quad v_{i}=\widetilde{v}_{i}+v_{i}^{\prime}
$$

as well as the viscous terms $\sigma_{i j}$ and $q_{i}$, are divided into their 'base flow' components $\bar{\rho}, \bar{p}, \tilde{h}, \tilde{v}_{i}, \bar{\sigma}_{i j}$, and $\bar{q}_{i}$, and into their 'residual' components $\rho^{\prime}, p^{\prime}, h^{\prime}, v_{i}^{\prime}, \sigma_{i j}^{\prime}$, and $q_{i}^{\prime}$. The former are required to satisfy the inhomogeneous Navier-Stokes equations

$$
D_{o} \bar{\rho}=0
$$

$$
D_{o} \bar{\rho} \tilde{v}_{i}+\frac{\partial \bar{p}}{\partial x_{i}}=\frac{\partial}{\partial x_{j}}\left(\tilde{T}_{i j}+\bar{\sigma}_{i j}\right)
$$

$$
D_{o} \bar{\rho} \tilde{h}_{o}-\frac{\partial \bar{p}}{\partial t}=D_{o} \tilde{H}_{o}+\frac{\partial}{\partial x_{j}}\left(\tilde{H}_{j}-\bar{q}_{j}+\tilde{v}_{i} \bar{\sigma}_{i j}\right)
$$


where the nonlinear operator $D_{o}$ is defined by

$$
D_{o} f \equiv \frac{\partial f}{\partial t}+\frac{\partial}{\partial x_{j}}\left(\widetilde{v}_{j} f\right)
$$

for any function $f$ and

$$
\widetilde{h}_{o} \equiv \widetilde{h}+\frac{1}{2} \widetilde{v}^{2}
$$

is the base flow stagnation enthalpy. The base flow variables, $\bar{\rho}, \bar{\rho}$, and $\widetilde{h}$ are also required to satisfy an ideal gas law equation of state,

$$
\widetilde{h}=c_{p} \widetilde{T}=\frac{c_{p}}{R} \frac{\bar{p}}{\bar{\rho}},
$$

and the 'sources strengths' $\widetilde{T}_{i j}, \widetilde{H}_{o}$, and $\widetilde{H}_{i}$, which are assumed to be localized within the jet, will be specified subsequently. The reason for using both over bars and tildes when defining the base flow components will become clear when equation (3.9) is introduced below.

To obtain the governing equations for the residual variables, substitute (2.4) into the Navier-Stokes equations (2.1) and subtract out (2.5) to obtain

$$
\begin{gathered}
D_{o} \rho^{\prime}+\frac{\partial m_{j}}{\partial x_{j}}=0, \\
D_{o}\left(m_{i}+\rho^{\prime} \widetilde{v}_{i}\right)+\frac{\partial}{\partial x_{j}} m_{j} \widetilde{v}_{i}+\frac{\partial p^{\prime}}{\partial x_{i}}=\frac{\partial}{\partial x_{j}} e_{i j}, \\
D_{o}\left(\pi+\mathbf{m} \bullet \widetilde{\mathbf{v}}+\frac{1}{2} \rho^{\prime} \widetilde{v}^{2}\right)-\frac{\partial p^{\prime}}{\partial t}+\frac{\partial}{\partial x_{j}} m_{j} \widetilde{h}_{o} \\
=-D_{o}\left(\frac{1}{2} \rho v^{\prime 2}+\widetilde{H}_{o}\right)-\frac{\partial}{\partial x_{j}}\left[\rho v_{j}^{\prime}\left(h^{\prime}+\frac{1}{2} v^{\prime 2}+\mathbf{v}^{\prime} \bullet \widetilde{\mathbf{v}}\right)+\widetilde{H}_{j}+q_{j}^{\prime}-\widetilde{v}_{i} \sigma_{i j}^{\prime}-v_{i}^{\prime} \sigma_{i j}\right],
\end{gathered}
$$


where $\mathrm{D}_{\mathrm{o}}$ (defined by (2.6)) is now a linear operator and we have put

$$
\pi \equiv \rho c_{p} T^{\prime}+\rho^{\prime} \tilde{h}=\frac{c_{p}}{R} p^{\prime}
$$

and

$$
\begin{gathered}
e_{i j} \equiv-\left(\rho v_{i}^{\prime} v_{j}^{\prime}+\widetilde{T}_{i j}-\sigma_{i j}^{\prime}\right), \\
m_{i} \equiv \rho v_{i}^{\prime}
\end{gathered}
$$

Equations ( $2.9 b$ and $c$ ) can be simplified by differentiating the quadratic terms on the left hand sides by parts and using equations (2.5a and $b),(2.9 a),(2.10)$, and (2.13b) (below) to obtain (Goldstein 1999, 2000, 2002)

$$
D_{o} m_{i}+\frac{\partial p^{\prime}}{\partial x_{i}}+m_{j} \frac{\partial \widetilde{v}_{i}}{\partial x_{j}}-\frac{\rho^{\prime}}{\bar{\rho}} \frac{\partial}{\partial x_{j}} \widetilde{\tau}_{i j}=\frac{\partial e_{i j}}{\partial x_{j}}
$$

$$
\frac{c_{v}}{R} D_{o} p^{\prime}+\frac{\partial m_{j} \widetilde{h}}{\partial x_{j}}+p^{\prime} \frac{\partial \widetilde{v}_{j}}{\partial x_{j}}-\frac{m_{i}}{\bar{\rho}} \frac{\partial \widetilde{\tau}_{i j}}{\partial x_{j}}=-D_{o}\left(\frac{1}{2} \rho v^{\prime 2}+\widetilde{H}_{o}\right)-\frac{\partial}{\partial x_{i}}\left(\rho v_{i}^{\prime} h_{o}^{\prime}+\widetilde{H}_{i}-\widetilde{T}_{i j} \widetilde{v}_{j}+q_{i}^{\prime}-v_{j}^{\prime} \sigma_{i j}\right)+e_{i j} \frac{\partial \widetilde{v}_{i}}{\partial x_{j}},(2
$$

where

$$
\tilde{\tau}_{i j} \equiv \delta_{i j} \bar{p}-\tilde{T}_{i j}-\bar{\sigma}_{i j}
$$

is the total base flow stress tensor and (in order to simplify the notation) we have defined $h_{0}^{\prime}$ by

$$
h_{o}^{\prime} \equiv h^{\prime}+\frac{1}{2} v^{\prime 2} .
$$

even though it is not equal to $h_{0}-\tilde{h}_{0}$. 
These equations can be put into a more transparent form by introducing the new dependent variables

$$
\begin{gathered}
p_{e}^{\prime} \equiv p^{\prime}+\frac{\gamma-1}{2} \rho v^{\prime 2}+(\gamma-1) \widetilde{H}_{o}, \\
u_{i}^{\prime} \equiv \frac{m_{i}}{\bar{\rho}}=\rho \frac{v_{i}^{\prime}}{\bar{\rho}}
\end{gathered}
$$

and source strengths

$$
\begin{gathered}
e_{i j}^{\prime} \equiv e_{i j}+\frac{\gamma-1}{2} \delta_{i j} \rho v^{\prime 2}=-\rho v_{i}^{\prime} v_{j}^{\prime}+\frac{\gamma-1}{2} \delta_{i j} \rho v^{\prime 2}+\sigma_{i j}^{\prime}, \\
\eta_{i}^{\prime} \equiv-\rho v_{i}^{\prime} h_{o}^{\prime}-q_{i}^{\prime}+\sigma_{i j} v_{j}^{\prime}, \\
\widetilde{e}_{i j} \equiv \widetilde{T}_{i j}-\delta_{i j}(\gamma-1) \widetilde{H}_{o},
\end{gathered}
$$

and

$$
\widetilde{\eta}_{i} \equiv \widetilde{H}_{i}-\widetilde{T}_{i j} \widetilde{v}_{j},
$$

to obtain

$$
\begin{gathered}
\bar{\rho} \frac{\bar{D}}{D t} \frac{\rho^{\prime}}{\bar{\rho}}+\frac{\partial}{\partial x_{j}} \bar{\rho} u_{j}^{\prime}=0, \\
\bar{\rho}\left(\frac{\bar{D}}{D t} u_{i}^{\prime}+u_{j}^{\prime} \frac{\partial \widetilde{v}_{i}}{\partial x_{j}}\right)+\frac{\partial}{\partial x_{i}} p_{e}^{\prime}-\frac{\rho^{\prime}}{\bar{\rho}} \frac{\partial}{\partial x_{j}} \widetilde{\tau}_{i j}=\frac{\partial}{\partial x_{j}}\left(e_{i j}^{\prime}-\widetilde{e}_{i j}\right),
\end{gathered}
$$

and 


$$
\frac{1}{\gamma-1}\left(\frac{\bar{D}}{D t} p_{e}^{\prime}+\gamma p_{e}^{\prime} \frac{\partial \widetilde{v}_{j}}{\partial x_{j}}+\gamma \frac{\partial}{\partial x_{j}} \bar{p} u_{j}^{\prime}\right)-u_{i}^{\prime} \frac{\partial \widetilde{\tau}_{i j}}{\partial x_{j}}=\frac{\partial}{\partial x_{j}}\left(\eta_{j}^{\prime}-\widetilde{\eta}_{j}\right)+\left(e_{i j}^{\prime}-\widetilde{e}_{i j}\right) \frac{\partial \widetilde{v}_{i}}{\partial x_{j}}
$$

where

$$
\frac{\bar{D}}{D t} \equiv \frac{\partial}{\partial t}+\widetilde{v}_{i} \frac{\partial}{\partial x_{i}}
$$

denotes the convective derivative in terms of the base flow velocity and $\gamma \equiv c_{p} / c_{v}$ is the specific heat ratio.

Equations (2.22) are the fundamental equations on which this paper is based. They are exact consequences of the original Navier-Stokes equations, but have been rearranged so that their left-hand sides are the same as the equations that would have been obtained by linearizing the convective form of the Euler equations about the base flow (2.5). In other words, they are the linearized inhomogeneous Euler (LIE) equations but with different (nonlinear) dependent variables $u_{i}^{\prime}$ and $p_{e}^{\prime}$, which should cause no particular difficulty since, for the present purpose, they can be treated as five linear equations in five unknowns-one of which reduces to the pressure fluctuations in the far field. The dependent variable $p_{e}^{\prime} /(\gamma-1)$ is closely related to the total residual energy density per unit volume. The right-hand sides correspond to the sources that would be obtained by imposing the external stress perturbation $e_{i j}^{\prime}-\widetilde{e}_{i j}$ and external energy flux perturbation $\eta_{i}^{\prime}-\widetilde{\eta}_{i}$. In other words, the fundamental equations (2.22) are just the linearized Navier-Stokes (LNS) equations with modified dependent variables and with the viscous stress perturbation replaced by the generalized Reynolds stress $e_{i j}^{\prime}-\widetilde{e}_{i j}$ and the heat flux perturbation replaced by the generalized stagnation enthalpy flux $\eta_{i}^{\prime}-\widetilde{\eta}_{i}$. A formal vector Greens function solution is given in the Appendix.

Lighthill $(1952,1954)$ pointed out that his equation implies that there is an exact analogy between the density fluctuations in any real flow and those produced by a quadrupole source in an ideal (non-moving) acoustic medium. The LIE (or LNS) equations (2.22) show that there is an exact analogy between the fluctuations occurring in any real flow and the linear inviscid fluctuations about an arbitrary flow produced by appropriate external stress and energy flux perturbations. The externally applied stress field can be associated with a quadrupole source in the stationary medium case considered by Lighthill. Unfortunately, there is no completely satisfactory definition of the 
multipole source order for a general moving medium, but one possible way of generalizing the stationary medium definition is to associate the term quadrupole with the source produced by a fluctuating stress field. The enthalpy flux term would then correspond to a dipole source. This is discussed in more detail in $\S 3.1$ below.

The classical acoustic analogy approaches referred to in the introduction follow the Lighthill philosophy and rearrange the Navier-Stokes equations into the form of a single moving medium wave-like equation with an appropriate source term. Unfortunately, the wave operator part of the resulting equation (usually the left-hand side) then contains both linear and nonlinear terms. Application of these equations to specific flows requires that this operator be linearized by moving the nonlinear terms to the right side, which causes the source term to become very complicated (see Colonius, Lele, and Moin 1997 and Freund and Fleischman 2002). The present approach differs from these classical approaches by carrying out an exact linearization at the outset and, as the LNS (or LIE) equations show, the resulting source terms are then quite simple and easy to interpret—provided the right set of dependent variables is selected. No additional rearrangement of the LIE equations is required when specific choices of the base flow are introduced.

One possible drawback of this approach is that it does not usually lead to a single wave equation (but see $\S 3.1$ below) for an appropriate dependent variable. This is not, however, really necessary and, as was first pointed out by Bechara et al. (1994), the primitive form of the equations (such as those being considered here) may even be more convenient for certain numerical purposes.

\section{Applications of fundamental equations}

In spite of these features, the LNS (or LIE) equations would still amount to little more than a sterile abstraction if specific base flow choices were not introduced. Some appropriate choices are discussed below.

\subsection{Homogeneous base flows}

We begin with the case where the base flow equations are homogeneous and inviscid so that the right sides of (2.5) are identically zero. A general class of solutions to these equations, which quite conveniently provides a good approximation to the actual mean flow field in a jet, is the unidirectional transversely sheared mean flow

$$
\widetilde{v}_{i}=\delta_{i 1} U\left(x_{2}, x_{3}\right), \quad \bar{p}=\text { constant }, \quad \bar{\rho}=\bar{\rho}\left(x_{2}, x_{3}\right)
$$


The continuity equation (2.22a) then decouples from the remaining four LNS (or LIE) equations, which now become

$$
\begin{gathered}
\bar{\rho}\left(\frac{\bar{D} u_{i}^{\prime}}{D t}+\delta_{i 1} u_{j}^{\prime} \frac{\partial U}{\partial x_{j}}\right)+\frac{\partial p_{e}^{\prime}}{\partial x_{i}}=\frac{\partial}{\partial x_{j}} e_{i j}^{\prime} \\
\frac{\bar{D} p_{e}^{\prime}}{D t}+\bar{p} \frac{\partial u_{j}^{\prime}}{\partial x_{j}}=(\gamma-1)\left(\frac{\partial \eta_{j}^{\prime}}{\partial x_{j}}+e_{1 j}^{\prime} \frac{\partial U}{\partial x_{j}}\right)
\end{gathered}
$$

where $\bar{D} / D t$ reduces to

$$
\frac{\bar{D}}{D t}=\frac{\partial}{\partial t}+U \frac{\partial}{\partial x_{1}}
$$

These equations are just the inhomogeneous compressible Rayleigh equations (Betchov and Criminale 1967) and, as is now well known (see chapter 1 of Goldstein 1976), the velocity variable $u_{i}^{\prime}$ can be eliminated between them (by taking the divergence of the first equation and the convective derivative of the second, subtracting the results and then using the first equation to eliminate the velocity fluctuation on the left-hand side) to obtain the inhomogeneous Pridmore-Brown equation

$$
L p_{e}^{\prime}=\frac{\bar{D}}{D t}\left(\frac{\partial}{\partial x_{i}} \widetilde{c}^{2} \frac{\partial e_{i j}^{\prime}}{\partial x_{j}}\right)-\frac{\partial U}{\partial x_{i}}\left(2 \widetilde{c}^{2} \frac{\partial^{2} e_{i j}^{\prime}}{\partial x_{1} \partial x_{j}}+(\gamma-1) \frac{\bar{D}^{2}}{D t^{2}} e_{1 i}^{\prime}\right)-(\gamma-1) \frac{\bar{D}^{2}}{D t^{2}} \frac{\partial \eta_{j}^{\prime}}{\partial x_{j}},
$$

where

$$
L \equiv \frac{\bar{D}}{D t}\left(\frac{\partial}{\partial x_{i}} \widetilde{c}^{2} \frac{\partial}{\partial x_{i}}-\frac{\bar{D}^{2}}{D t^{2}}\right)-2 \frac{\partial U}{\partial x_{j}} \frac{\partial}{\partial x_{1}} \widetilde{c}^{2} \frac{\partial}{\partial x_{j}}
$$

is the variable density Pridmore-Brown (1958) operator and 


$$
\widetilde{c}^{2}=\bar{p} / \bar{\rho}\left(x_{2}, x_{3}\right)
$$

is the square of the mean flow sound speed. This is yet another form of Lilley's equation with a quadrupole/dipole type source term, which is clearly more complicated than the original Rayleigh equation sources.

This equation does not reduce to Lighthill's result in the limit $U \rightarrow 0$ and $\bar{\rho} \rightarrow$ constant considered by Lighthill (1952) but it does, with some rearrangement, reduce to a form of Lighthill's equation obtained by Lilley (1974).

Lighthill originally suggested that the inconvenient $\delta_{i j}\left(p-c_{o}^{2} \rho\right)$ term that appears in his stress tensor should be associated with non-isentropic density fluctuations, which are presumably produced by viscous effects and are, therefore, likely to be negligible. Lilley (1974) pointed out that this term also has an isentropic component (which may be an important noise source when the mean density (i.e., temperature) is non-constant) and proceeded to derive an equation, which separated out these two components. Unfortunately, the resulting source term contains a dipole component (corresponding to the enthalpy flux $\eta_{i}^{\prime}$ in equation (3.3)). However, the second time derivative that appears in (3.6) causes it to have the same efficiency as the quadrupole source term in the low-frequency (or long wavelength) limit—, which is the only situation where the multipole nature of the source has any significance.

It is worth noting that the dependent variables $v_{i}^{\prime}$ and $h^{\prime}$ and consequently the 'source strengths' $e_{i j}^{\prime}$ and $\eta_{i}^{\prime}$ that appear in (3.2) and (3.3) do not have zero time averages and therefore still contain an undesirable mean flow component. It is also worth noting that these equations possess homogeneous solutions corresponding to spatially growing instability waves on the base flow (3.1) (Betchov and Criminale, 1967). They also possess homogeneous solutions corresponding to the continuous spectrum. The complete solution to these equations consists of a particular solution plus these homogeneous contributions, but the result is meaningless because the instability waves become unbounded (infinite) far downstream in the flow. The usual resolution is to completely neglect the instability wave contribution. Dowling, Ffowcs Williams, and Goldstein (1978) attempted to justify this by invoking causality arguments.

\subsection{Steady non-parallel base flow}

A better resolution is obtained by choosing the base flow to be the actual mean flow of the jet. The over bar on the dependent base flow variables would then denote the time average 


$$
\bar{\bullet} \equiv \lim _{T \rightarrow \infty} \frac{1}{2 T} \int_{-T}^{T} \bullet(\mathbf{x}, t) \mathrm{d} t
$$

where the dot is a place holder for $\rho, v_{i}, p$, and $h$, and

$$
\widetilde{\bullet} \equiv(\overline{\rho \bullet} / \bar{\rho})
$$

denotes a Favre averaged quantity (Lele 1994) for all variables except $\tilde{h}_{o}$, which is defined by (2.7). Notice that (2.8) is completely consistent with the overall ideal gas law (2.3) when the tilde is defined in this fashion.

The time derivatives drop out of the base flow equations (2.5), which do not, of course, form a closed system. The source strengths are given by

$$
\begin{gathered}
\widetilde{T}_{i j}=-\bar{\rho}\left(\widetilde{v_{i} v_{j}}-\widetilde{v}_{i} \widetilde{v}_{j}\right), \\
\widetilde{H}_{o}=\frac{1}{2} \widetilde{T}_{i i}, \\
\widetilde{H}_{j}=-\bar{\rho}\left(\widetilde{h}_{o} v_{j}-\widetilde{h}_{o} \widetilde{v}_{j}\right)-\widetilde{H}_{o} \widetilde{v}_{j},
\end{gathered}
$$

The base flow equations are now the ordinary RANS equations, which are usually closed by assuming some sort of model relating the source terms to the mean flow variables $\widetilde{v}_{i}, \bar{\rho}, \bar{p}$, and $\tilde{h}$ and their derivatives, such as the Boussinesq model (Speziale 1991; Speziale and So 1998)

$$
\widetilde{T}_{i j}=\mu_{T}\left(\frac{\partial \widetilde{v}_{i}}{\partial x_{j}}+\frac{\partial \widetilde{v}_{j}}{\partial x_{i}}-\frac{2}{3} \frac{\partial \widetilde{v}_{k}}{\partial x_{k}} \delta_{i j}\right)+\frac{2}{3} \widetilde{H}_{o} \delta_{i j}
$$

for the Reynolds stresses, with additional equations to determine the turbulent viscosity $\mu_{T}$ and a similar model for $\widetilde{H}_{j}$. The fundamental LNS equations (2.22) remain exact even when these approximations are introduced since, as 
already noted, the base flow source strengths can be arbitrarily specified. But, (3.8) would only be an approximation and the base flow would be somewhat different from the actual mean flow in the jet.

The time average flow need not, of course, be determined from a RANS computation. It can, for example, be determined by applying (3.8) to a LES or a DNS computation. The base flow sources (3.10) and (3.12) can then be written more compactly as

$$
\begin{gathered}
\widetilde{T}_{i j}=-\bar{\rho} \widetilde{v_{i}^{\prime} v_{j}^{\prime}}, \\
\widetilde{\eta}_{i} \equiv \widetilde{H}_{i}-\widetilde{T}_{i j} \widetilde{v}_{j}=-\bar{\rho} \widetilde{h_{o}^{\prime} v_{i}^{\prime}}
\end{gathered}
$$

with $\widetilde{H}_{o}$ still be given by (3.11) and $h_{0}^{\prime} \neq h_{0}-\tilde{h}_{0}$ is given by (2.15). The inviscid LIE (or LNS) equation source strengths $e_{i j}^{\prime}-\widetilde{e}_{i j}$ and $\eta_{i}^{\prime}-\widetilde{\eta}_{i}$ would be proportional to the differences between the fluctuating and Favre averaged Reynolds stresses and enthalpy fluxes, which means that these sources would now be true fluctuating quantities with zero time averages, even in the viscous case. This insures that the residual variables will have that property as well. (The fact that $\overline{p_{e}^{\prime}}=\overline{u^{\prime}}=0$ can also be verified directly from (2.16), (2.17), (3.11), and (3.14).)

The corresponding LIE equation sources, which are in principle exact and (as noted in section 2) simpler than those that appear in the literature, must still be modeled. This is most easily accomplished by using the formal Greens function solution (A-9) to express the far field pressure in terms of the near field source distribution. The result can then be time averaged to relate the mean square pressure in the far field to the source correlation function, which means that it is only necessary to model this latter quantity and not the instantaneous sources themselves. The resulting fourth order two point time delayed correlations involve pure fluctuating quantities and do not contain the awkward mean flow component that appears in the parallel base flow model discussed in $\S 3.1$. (The importance of this property was demonstrated by Bogey, Bailly, and Juvé (2002)). These correlations can be expressed in terms of second order velocity and enthalpy correlations (which are very close to what is experimentally measured) by invoking the quasi-normal approximation (Millionschikov, 1941) — which is thought to be fairly accurate in this context.

The most important difference between the present results and the parallel flow results of $\S 3.1$ is that the homogeneous solutions to the LIE (or LNS) equations, which presumably correspond to instability waves growing 
and then decaying on the diverging non-parallel base flow, will always remain bounded. This eliminates the paradox that arose in $\S 3.1$.The complete LIE equation solution therefore remains bounded and can, as a consequence, be used to calculate the radiated sound. It consists of a particular solution that is driven by the sources (i.e. it satisfies causality and therefore the appropriate upstream boundary conditions) and a homogeneous solution that is driven by the upstream boundary conditions, say at the nozzle exit.

The significance of this result can be understood by considering an idealized subsonic or a nearly perfectly expanded supersonic jet. The spread rate, say $\varepsilon$, of the base flow will then be uniformly small, which implies that the mean pressure gradient will be $0\left(\varepsilon^{2}\right)$, the mean cross-flow will be $0(\varepsilon)$, and all streamwise changes in the base flow will occur on the slow streamwise length scale $\varepsilon x_{1}$. The source driven particular solution can, as noted above, be constructed from the casual vector Green function, which satisfies (A-7) and consists of a component that decays to zero when the unscaled streamwise coordinate $x_{1}-x_{1} \bullet$ becomes large plus a component that becomes unbounded when the unscaled streamwise coordinate becomes large but decays to zero on the long (slow) stream-wise length scale $\varepsilon\left(x_{1}-x_{1} \bullet\right)$. The former component can be calculated by treating the slow variable $\mathcal{E} \mathrm{x}_{1}$ as a parameter and using the parallel flow solution (3.1) to simplify the linearized Euler operator (A-1).

This approximation cannot, however, be used to determine the latter component (which corresponds to a superposition of linear instability waves, Briggs, 1964; Bers, 1975), since the resulting local solution would become unbounded and would not remain valid over the long streamwise length scale $\varepsilon\left(x_{1}-x_{1} \bullet\right)$ on which this component evolves. The appropriate result is obtained by retaining $\mathrm{O}(\varepsilon)$ terms in the linearized Euler operator (A-1) and using the multiple scales method together with the WKBJ approximation to render the solution uniformly valid.

Equation (A-8) (or (A-9) and (A-10)) shows that both components of the Greens function act on the same source term. Each component is capable of producing acoustic radiation - even at subsonic Mach numbers (Tam and Morris 1980). The first corresponds to the usual Lilley equation solution but with slowly varying coefficients and with $e_{i j}^{\prime}$ and $\eta_{i}^{\prime}$ replaced by $e_{i j}^{\prime}-\widetilde{e}_{i j}$ and $\eta_{i}^{\prime}-\widetilde{\eta}_{i}$, respectively. The second is associated with linear instability waves but is very different from conventional instability models, since these waves are now continuously generated along the length of the jet and do not constitute separate sound sources. Their only role is to provide another path for propagating the source generated pressure fluctuations to the far field.

Each Greens function component can be thought of as a filter that only allows certain elements of the source spectrum to reach the far field. Since these components are expected to respond to different portions of this spectrum, the resulting radiation field should exhibit a bi-model structure. Tam, Golebiowski, and Seiner (1996) 
recently presented some experimental evidence which strongly suggests that the jet sound field actually has such a structure - especially at supersonic Mach numbers. The contribution of the second Greens function component will probably be fairly small at subsonic Mach numbers but is likely to be dominant at supersonic speeds. The present result is therefore very different than the parallel flow model of the previous subsection.

The homogeneous solution, which is usually associated with the large-scale coherent structures (Crow and Champagne 1971; Mankbadi and Liu 1984; Gaster, Kit, and Wygananski 1985), is similar to the second component of the Greens function solution in that it involves instability waves and must also be determined from a multiple scales analysis. It is, however, expected to be small compared to the contribution of that component, since it is only generated at the upstream boundary while the latter is generated over an extended streamwise region. However, the only unequivocal distinction between the two solutions is that the homogeneous solution generates sound through a linear process while the particular solution generates sound through non-linear mechanisms. They can, of course, be coupled since the acoustic radiation from the particular solution can trigger instability waves in the upstream region, say at the nozzle lip. But, this effect is probably insignificant (relative to the effect of the pre-existing upstream disturbances) for the noisy flows that are of technological interest.

Another difference between the present results and those of the previous subsection is the $\tilde{\tau}_{i j}$ term on the lefthand side of $(2.22 b)$, which drops out of the parallel base flow model of $\S 3.1$ but can now produce a potentially strong coupling between $(2.22 b)$ and $(2.22 c)$ and the continuity equation $(2.22 a)$. This term is expected to be small when the mean flow is nearly parallel, as it is in idealized subsonic jets. However, real turbojet flows are not all that parallel in the vicinity of the nozzle — especially when the latter is of the mixer-ejector type. The term could also be important for improperly expanded supersonic jets and in flows that arise in non-aeronautical applications of the LNS equations (2.22).

\subsection{Unsteady compressible base flows}

While the coherent structures are at best poorly defined, there is some evidence to suggest that they may not be adequately represented by the linear instability waves. A better representation can be obtained by making them part of the base flow, which would then, of course, have to be unsteady. Calculations based on linear stability theory (Reau and Tumin 2000a and b; Liu 1998) suggest that good results can be obtained by allowing the base flow momentum source $\widetilde{T}_{i j}$ to again be of the Boussinesq form (3.13) with a similar representation for the enthalpy source $\widetilde{H}_{j} \cdot \widetilde{H}_{o}$ would still be given by (3.18). The RANS type eddy viscosity model also seems to work fairly 
well. The resulting base flow equations would then be the unsteady RANS or (URANS) equations with the unsteadiness being driven by the random upstream boundary conditions. The LNS (or LES) equations (2.22) are again exact.

Bastin et al. (1997) carried out the relevant computation for a two dimensional planar jet. They used a standard $\mathrm{k}-\varepsilon$ model for the eddy viscosity with an appropriately reduced numerical coefficient. Their results seem to over predict the contributions of the large scale structures, which suggests that a more refined turbulence model might be in order.

The relation between the URANS solutions and the coherent structures can be made more plausible by letting the over bars on the base flow variables denote the ensemble/phase average

$$
\bar{f}=\lim _{N \rightarrow \infty} \frac{1}{N M} \sum_{j=1}^{M} \sum_{i=1}^{N} f_{i}\left(\mathbf{x}, t+t_{i j}\right)
$$

where $f$ can be any of the dependent variables, $f_{i}$ denotes the value of $f$ in the $i^{\text {th }}$ realization of the flow and the $t_{i j}$ denote appropriate time delays, which are introduced to account for the fact that each of the $\mathrm{M}$ coherent structures will have a different phase in each realization of the flow. Bastin et al. (1997) proposed using a conventional ensemble average to represent the coherent structures, which would correspond to setting $t_{i j}=0$ in. (3.16). Equation (3.16) will represent a conventional phase average when $M=1$ and all of the members of the ensemble correspond to the same realization of the flow. This would probably provide a good representation of the coherent structures in a flow that is artificially excited by a periodic source (Liu, 1998). The appropriate time intervals $t_{i l}$ would then be integral multiples of the period $\mathrm{T}$ of the excitation. The precise values of the $t_{i j}$ would, of course, be unknown in naturally occurring flows, but that is largely irrelevant for the present purposes. $\bar{f}$ consists of the time average of $f$ (i.e., the mean flow) plus the ensemble/phase average of the fluctuating component of $f$ (Reynolds and Hussain 1970; Hussain 1983).

The tilde is still defined by equations (2.8) and (3.9), the base flow source terms $\widetilde{T}_{i j}, \widetilde{H}_{o}$, and $\widetilde{H}_{i}$ are still given by (3.10) to (3.12) (but not by (3.14) and (3.15)) and the base flow is no longer non-radiating. It is likely to be composed of the larger scale components of the motion and may therefore bear some resemblance to the resolved component of a corresponding Large Eddy Simulation (LES) (Bastin et al. 1997). As in LES, the sources, which now account for the effect of the random turbulence on the coherent motion, must be modeled. Using the 
Boussinesq type model (3.13) for the momentum source (3.10) and a similar model for the enthalpy source (3.12) leads to the URANS equations alluded to above.

While still expensive, URANS computations are much less demanding of computer resources than the DNS computations referred to in the INTRODUCTION (Bastin et al. 1997). There is, therefore, some hope that they can be used to predict the sound emission from realistic flows. In any event, the residual variables (which now correspond to the incoherent part of the motion) can, and probably do, contain significant large scale components, which would not be the case in LES where the pure spatial filtering that is usually used would cut off these components. These variables are again governed by the LIE (or LNS) equations (2.22) but the homogeneous solution (if it exists) must now be set to zero, since the upstream boundary conditions that would drive this solution are already satisfied by the base flow. This approach formally resembles the two stage LES approach proposed by Bodony and Lele (2002), but the philosophical basis and modeling assumptions are quite different.

The complete sound field can, at least in principle, be calculated by adding the base flow sound field to the far-field LIE (or LNS) equation solution. The latter equations are, in principle, exact, but their source terms still have to be modeled .It is, however, no longer possible to use the procedure described in the previous subsection to relate the far-field pressure correlation to the correlation of the source function since the base flow is now unsteady. It is therefore necessary to model the instantaneous source strengths rather than their correlations-which is a much more difficult task. It may be possible to use some sort of stochastic source model such as the ones used by Bailly et al. (1995) and Bodony and Lele (2002). A more expedient approach might be to neglect the contribution of the unsteady coherent component of the base flow to the coefficients of the LIE equation operator (A-1), since it is likely to be fairly small compared to the mean flow contribution. The incoherent far-field pressure auto-correlation could then be related to the source correlations, which would now, however, involve only the incoherent part of the motion. But this creates somewhat of a dilemma because only the correlation of the total unsteady motion is actually measured in the experiments. And even if this difficultly could be overcome, the result would only be meaningful if the coherent and incoherent components of the motion were relatively uncorrelated (Bastin et al 1997). It would then be a generalization of the RANS equation approach described in the previous subsection and would reduce to that approach if the base flow computation were linearized about the mean flow and source term perturbations were neglected.

However, we have already indicated that this linearization may be unrealistic and Reau and Tumin (2002a and b) show that the perturbations in the source terms cannot be neglected. Liu (1988) and Mankbadi and Liu (1984) 
adopted an intermediate approach in which the coherent motion was determined from integral conservation laws by using an approximate shape functions taken from linear instability theory.

The base flow equations with source strengths (3.10) to (3.12) formally resemble the filtered Navier-Stokes equations (Rogallo and Moin, 1984) and there are a number of interesting ways of choosing the filter in these equations. Goldstein (2002) showed that it can even be chosen to only pass wave number components with subsonic phase velocity in the stream-wise direction* which, according to conventional wisdom (see, for example, Freund 2001), should cause the unsteady base flow to be non-radiating. But this probably needs further study. The relevant flow can be computed by using a Fourier-Spectral method with only subsonically convecting spectral base functions retained in the computation. The advantage of using an unsteady but non-radiating base flow is discussed in the next subsection.

\subsection{Non-radiating unsteady base flows}

The radiated sound is typically many orders of magnitude smaller than the non-radiating component of the motion in virtually all high speed air jets, as well as in most other high speed flows. In fact, it is usually many orders of magnitude smaller than the errors incurred in computing the non-radiating part of the flow. A number of investigators have even suggested that the 'acoustic component' of the numerical solution to the full Navier-Stokes equations would be hopelessly corrupted by these errors or even by the computational noise itself (e.g. Roe 1992; Crighton 1993). This difficulty would certainly be overcome if the radiating component of the motion could be separated out and calculated as if it were a small perturbation about the non-radiating component, which amounts to requiring that the base flow in the decomposition (2.4) be the entire (or almost the entire) non-radiating component

\footnotetext{
*There is a subscript 1 missing from the $\mathrm{k}$ on the right side of equation [49] of that reference. of the motion.
} 
A nice feature of the RANS base flow of $\S 3.2$, or for that matter, any steady base flow, is that it is nonradiating. Another possible non-radiating base flow is an incompressible flow. More generally, the base flow can always be made non-radiating by requiring that

$$
\frac{\partial \bar{\rho}}{\partial \mathrm{t}}=\mathrm{O}\left(|x|^{-4}\right) \text { as }\left|x_{\perp}\right| \rightarrow \infty,
$$

where $\boldsymbol{x}_{\perp}$ is the transverse coordinate vector. This will force the flow to behave incompressibly at large transverse distances and should therefore prevent any acoustic radiation from reaching the far-field.

It is, in general, impossible to satisfy the homogenous energy equation $(2.5 c)$ and still impose arbitrary requirements on $\partial \bar{\rho} / \partial t$. We can, however, choose the energy equation sources $\widetilde{H}_{o}$ and $\widetilde{H}_{i}$ so that this equation is identically satisfied for any $\bar{\rho}$ by setting

$$
(\gamma-1) \tilde{H}_{0}=\bar{p}_{e} \equiv \bar{p}+\frac{\gamma-1}{2} \bar{\rho} \tilde{v}^{2}, \quad \tilde{H}_{i}=\bar{p} \tilde{v}_{i}+\bar{q}_{i}-\bar{\sigma}_{i j} \tilde{v}_{j}
$$

The momentum equation source strength $\widetilde{T}_{i j}$ can again be set to zero. It is then possible to preselect $\bar{\rho}$ and require that is satisfy (3.17). This means that (2.5a) and (2.5b) are similar to the incompressible Navier-Stokes equations in that they can be solved for $\widetilde{v}_{i}$ and $\bar{p}$ independently of the energy equation.

The source terms in the LNS equations $(2.22 b)$ and $(2.22 c)$ now become

$$
\begin{gathered}
\mathrm{e}_{\mathrm{ij}}^{\prime}-\widetilde{\mathrm{e}}_{\mathrm{ij}}=-\rho\left(v_{\mathrm{i}}^{\prime} v_{\mathrm{j}}^{\prime}-\frac{\gamma-1}{2} \delta_{\mathrm{ij}} v^{\prime 2}\right)+\sigma_{\mathrm{ij}}^{\prime}+\delta_{\mathrm{ij}}\left(\overline{\mathrm{p}}+\frac{\gamma-1}{2} \bar{\rho}^{2}\right) \\
\eta_{i}^{\prime}-\widetilde{\eta}_{i}=-\rho v_{i}^{\prime} h_{o}^{\prime}-\bar{p} \widetilde{v}_{i}-q_{i}+\sigma_{i j} v_{j}-\sigma_{i j}^{\prime} \widetilde{v}_{j}
\end{gathered}
$$

A cleaner result is obtained when $\widetilde{H}_{o}$ is eliminated from the residual equation dependent variable $p_{e}^{\prime}$ by letting

$$
\mathrm{p}_{\mathrm{e}}^{\prime} \rightarrow \mathrm{p}_{\mathrm{e}}^{\prime}-(\gamma-1) \widetilde{\mathrm{H}}_{\mathrm{o}}=\mathrm{p}^{\prime}+\frac{(\gamma-1)}{2} \rho v^{\prime 2}
$$


The left sides of the LNS (or LIE) equations (2.22) remain unchanged and their right-hand sides become

$$
\begin{gathered}
\operatorname{RHS}(2.22 \mathrm{a})=0 . \\
\operatorname{RHS}(2.22 \mathrm{~b})=\frac{\partial e_{i j}^{\prime}}{\partial x_{j}},
\end{gathered}
$$

and

$$
\operatorname{RHS}(2.22 \mathrm{c}) \rightarrow-\frac{1}{\gamma-1}\left(\frac{\overline{\mathrm{D}}}{\mathrm{Dt}} \overline{\mathrm{p}}+\gamma \overline{\mathrm{p}} \frac{\partial \widetilde{v}_{\mathrm{j}}}{\partial \mathrm{x}_{\mathrm{j}}}\right)+\frac{\partial\left(\eta_{\mathrm{j}}^{\prime}-\widetilde{\mathrm{q}}_{\mathrm{j}}\right)}{\partial \mathrm{x}_{\mathrm{j}}}+\left(\mathrm{e}_{\mathrm{ij}}^{\prime}+\widetilde{\sigma}_{\mathrm{ij}}\right) \frac{\partial \widetilde{v}_{\mathrm{i}}}{\partial \mathrm{x}_{\mathrm{j}}}
$$

The strictly incompressible base flow is recovered by setting $\bar{\rho}=$ constant. Hardin and Pope (1994) developed a two-stage theory in which the base flow was incompressible and viscous but the residual flow was homentropic. A more consistent approximation would be to set the residual viscous and heat conduction terms to zero in ( $3.21 \mathrm{~b}$ and c), which roughly corresponds to the somewhat weaker requirement that the residual flow be isentropic.

When the flow is inviscid and the base flow density $\bar{\rho}$ is constant, the deviation $v_{i}^{\prime}$ of the velocity form and the incompressible approximation $\widetilde{v}_{i}$ should be $0\left(M^{2}\right)$ when the Mach number $M$ is small and the quadratic source terms $\partial\left(e_{i j}^{\prime} / \partial x_{j}\right)$ and $\left(\partial \eta_{j}^{\prime} / \partial x_{j}\right)+e_{i j}^{\prime}\left(\partial \widetilde{v}_{i} / \partial x_{i}\right)$ should then be negligible compared to the base flow source term in (3.21 c). This implies that the sound field is primarily generated by the convective derivative of the incompressible pressure fluctuations, since $\partial \widetilde{v}_{i} / \partial x_{i}=0$ in this case. The result is, therefore, related to Ribner's (1962) 'dilitation' theory of jet noise, but unlike Ribner's original proposal, the interaction of the sound with the incompressible flow is now explicitly accounted for. Also, the purely convected pressure disturbances (i.e., the pseudo sound) are now (explicitly) non-radiating. The important thing is that the sound sources are nearly independent of the residual variables, which should certainly be required if the latter are to be associated with the acoustic part of the motion. Tam and Auriault (1999) have also proposed using the pressure fluctuation (though not the incompressible pressure fluctuations) as a sound source.

The strictly incompressible approximation cannot remain valid at supersonic Mach numbers (even for ideally expanded jets), since shear layer spread rates and turbulence structures are known to be Mach number dependent in 
this case (Smits and Dussauge 1996, ch. 6), and it is even questionable at moderately high, say above 0.3, subsonic Mach numbers. It may, however, be possible to choose the density $\bar{\rho}$ so that the base flow $(2.5 a)$ and $(2.5 b)$ with $\widetilde{T}_{i j}=0$ remains close to the real flow and it still non-radiating (i.e., it satisfies (3.17)). An intuitively appealing way of accomplishing this is to require that $\bar{\rho}$ be convected by a localized flow field, say $\widetilde{V}_{i}(\mathbf{x}, t)$, whose streamlines are constrained to lie within the interior of the jet, which means that

$$
\frac{\partial}{\partial \mathrm{t}}\left(\frac{\bar{\rho}}{\overline{\mathrm{R}}}\right)+\widetilde{\mathrm{V}}_{\mathrm{j}} \frac{\partial}{\partial \mathrm{x}_{\mathrm{j}}}\left(\frac{\bar{\rho}}{\overline{\mathrm{R}}}\right)=0
$$

where $\bar{R}$ becomes constant at large distances from the flow, but is otherwise arbitrary. The upstream boundary conditions can then be chosen to insure that $\bar{\rho}$ decays at large transverse distances from the flow, which should then ensure that (3.17) is satisfied. This can be thought of as a generalization of Morkovin's hypothesis (Morkovin 1962; Bradshaw 1978) which, unlike the original proposal allows for fluctuations in the density. There is therefore some hope that this model (unlike the original Morkovin proposal) will provide a reasonable approximation for jet-like flows, even at supersonic Mach numbers (Smits and Dussauge 1996, p. 138).

$\bar{\rho}$ and $\widetilde{V}_{i}$ will also satisfy an equation of the form

$$
\frac{\partial}{\partial \mathrm{t}} \bar{\rho}+\frac{\partial}{\partial \mathrm{x}_{\mathrm{j}}} \bar{\rho} \widetilde{\mathrm{V}}_{\mathrm{j}}=0
$$

if $\widetilde{V}_{i}$ and $\bar{R}$ are required to satisfy the steady flow type continuity equation

$$
\frac{\partial}{\partial x_{j}} \bar{R} \widetilde{V}_{j}=0
$$

In which case, the continuity equation (2.5a) becomes 


$$
\frac{\partial}{\partial \mathrm{x}_{\mathrm{i}}} \bar{\rho}\left(\widetilde{v}_{\mathrm{i}}-\widetilde{\mathrm{V}}_{\mathrm{i}}\right)=0
$$

which means that the base flow satisfies the steady continuity equation in a reference frame moving with the velocity $\widetilde{V}_{i}$, i.e., the relative momentum flux $\bar{\rho}\left(\widetilde{v}_{i}-\widetilde{V}_{i}\right)$ now has zero divergence.

For numerical purposes it may be better to determine $\bar{\rho}$ from an equation of the form

$$
\frac{\partial \bar{\rho}}{\partial \mathrm{t}}=-\frac{\partial^{2} \widetilde{\mathrm{S}}_{\mathrm{ij}}}{\partial \mathrm{x}_{\mathrm{i}} \partial \mathrm{x}_{\mathrm{j}}}
$$

where $\widetilde{S}_{\mathrm{ij}}(\mathrm{x}, \mathrm{t})$ is required to vanish at an appropriate rate as $x \rightarrow \infty$, but is otherwise arbitrary. This can be seen by eliminating the momentum flux $\bar{\rho} \widetilde{v}_{i}$ between the base flow continuity and momentum equations $(2.5 a)$ and $(2.5 b)$ to obtain the Poisson's equation

$$
\nabla^{2} \bar{p}=\frac{\partial^{2} S_{\mathrm{ij}}^{*}}{\partial x_{\mathrm{i}} \partial x_{\mathrm{j}}} \equiv-\frac{\partial^{2}\left(\bar{\rho} \widetilde{v}_{\mathrm{i}} \widetilde{v}_{\mathrm{j}}-\dot{\tilde{S}}_{\mathrm{ij}}+\widetilde{\sigma}_{\mathrm{ij}}\right)}{\partial x_{\mathrm{i}} \partial x_{\mathrm{j}}} .
$$

where the dot denotes differentiation with respect to time. The free-space Greens function solution to this equation is

$$
\bar{p}(x, t)=\frac{1}{4 \pi} \frac{\partial^{2}}{\partial x_{\mathrm{i}} \partial x_{\mathrm{j}}} \int \frac{S_{\mathrm{ij}}^{*}\left(x^{\prime}, t\right)}{\left|x^{\prime}-x\right|} d x^{\prime}
$$

which shows that $\bar{p}=0\left(|\mathbf{x}|^{-3}\right)$ as $|\mathbf{x}| \rightarrow \infty$ if $\widetilde{S}_{i j}$ is chosen so that $S_{i j}^{*}=o\left(|\mathbf{x}|^{-3}\right)$ as $|\mathbf{x}| \rightarrow \infty$. This is the same as the pressure decay rate experienced by a localized incompressible flow (Crow 1970) and is therefore likely to be fast enough to greatly simplify the implementation of the far-field boundary conditions. The density $\bar{\rho}$ will still satisfy (3.23) (and therefore (3.25)) if $\widetilde{V}_{i}$ is defined by 


$$
\widetilde{\mathrm{V}}_{i} \equiv \frac{1}{\bar{\rho}} \frac{\partial \widetilde{\mathrm{S}}_{\mathrm{ij}}}{\partial \mathrm{x}_{\mathrm{j}}} .
$$

The density can easily be localized if $\widetilde{V}_{i}$ is required to be steady. It could even be taken to be the actual mean velocity of the jet, as determined from a preliminary RANS calculation (see § 3.2). In which case, (3.24) would require that $\bar{R}$ be the mean density of the jet.

There are, of course, many other possible choices for $\bar{R}$ and $\widetilde{V}_{i}$. They could, for example, be chosen so that the base flow source term

$$
-\frac{1}{\gamma-1}\left(\frac{\bar{D}}{D t} \bar{p}+\gamma \bar{p} \frac{\partial \widetilde{v}_{j}}{\partial x_{j}}\right)
$$

in (3.21c) has zero mean, which would then ensure that the base flow satisfies the correct homogeneous energy equation in the mean and that $p_{e}^{\prime}$ has zero mean. This would, however, require that an additional integraldifferential equation be solved along with (2.5a and $b$ ), which would make the base flow computation more difficult—possibly more difficult than an actual compressible DNS computation, which would, of course, defeat the whole purpose of the two stage approach.

Lighthill (1952) argued that the strength of his quadrupole source could be obtained to a good approximation by calculating its value for an equivalent flow devoid of sound. The present result provides an analytical basis for that idea. It is, of course, possible to move the residual stresses to the left side of the LNS equations and calculate the sound from the full nonlinear equations, but that would make the present approach more complicated and computationally more expensive than solving the original Navier-Stokes equations. In fact, the main justification for using this approach is to ensure that the errors incurred in computing the non-radiating component of the motion do not unduly contaminate the radiating component — which is best accomplished can only be accomplished if the residual variables are required to be much smaller than the base flow variables, which should ensure that the linearization is justified. 


\section{Conclusions}

A general set of linearized inhomogeneous Euler (LIE) equations has been derived and used to put a number of classical and recently proposed noise prediction methods on a more rational basis and in most cases extend them in new directions. The source terms are exactly the same as those that would be obtained by imposing externally applied stress and energy flux perturbations on the flow and the equations are therefore exactly the same as the linearized Navier-Stokes (LNS) equations, but with the viscous stress perturbation replaced by an appropriate Reynolds stress and the heat flux perturbations replaced by an appropriate enthalpy flux. The dependent variables are also somewhat different.

Lighthill pointed out that his equation implied that there was an exact analogy between the density fluctuations in any real flow and those produced by a quadrupole source in an ideal (non-moving) acoustic medium. The LNS (or LIE) equations show that there is an exact analogy between the fluctuations in any real flow and the linear inviscid fluctuations about an arbitrary base flow produced by externally imposed stress and energy flux perturbations. This does not, of course, imply that the LNS (or LIE) equations, or for that matter, any of the previously proposed 'acoustic analogy' equations can provide an unambiguous identification of sources, except perhaps for the generalized incompressible flows considered in $§ 3.4$. These equations are only useful when the 'base flow' is reasonably close to the actual fluid motion and, in most cases, can only serve as a guide for identifying and ultimately modeling the apparent sources of sound. The sound is just a by-product of all the processes occurring in the flow and it is highly unlikely that "true" sound sources can be identified in any realistic turbulent flow.

The LIE equations are made explicit by considering various possible base flows. A number of such flows, corresponding to progressively more complex choices of the base flow source terms, were described in $\S 3$. The first three choices require source term modeling as in the classical Lighthill approach. But these terms now generate only part of the sound field in at least two of these. The remainder must be determined by the direct solution of the appropriate linear or non-linear equations, which is another indication that the mathematical sources need not be the true sources of sound. The last subsection considers formulations in which the dominant LNS equation source term can be computed but require that the base flow be chosen to minimize the neglected source terms.- - which is a different approach to jet noise modeling that has yet to be exploited. 


\section{Appendix}

The five LNS equations (2.22) can be written more compactly by introducing the five dimensional operator

$$
L_{\mu v} \equiv \delta_{\mu v} D_{o}+(\gamma-1) \delta_{v 4} \partial_{\mu}+\partial_{\nu}\left(\frac{\widetilde{c}^{2}}{\gamma-1} \delta_{\mu 4}+\delta_{\mu 5}\right)+K_{\mu v}
$$

where $\mu, v=1,2, \ldots, 5$ while the Latin indices $i, j$ are restricted to the range 1,2,3.

$$
\begin{gathered}
K_{\mu \nu} \equiv \partial_{\nu} \widetilde{v}_{\mu}-\frac{1}{\bar{\rho}} \frac{\partial \widetilde{\tau}_{\mu j}}{\partial x_{j}} \delta_{v 5}+\left((\gamma-1) \frac{\partial \widetilde{v}_{j}}{\partial x_{j}} \delta_{\nu 4}-\frac{1}{\bar{\rho}} \frac{\partial \widetilde{\tau}_{v j}}{\partial x_{j}}\right) \delta_{\mu 4} \\
\partial \mu \equiv\left\{\begin{array}{l}
\frac{\partial}{\partial x_{i}}, i=\mu=1,2,3 \\
0, \text { otherwise }
\end{array}\right.
\end{gathered}
$$

$\widetilde{v}_{\mu}$ and $\tau_{\mu j}$ are both zero when $\mu>3, \widetilde{c}^{2}$ is given by (3.7), the Einstein summation convention is still being used and $\delta_{\mu v}$ is the five dimensional Kronecker delta.

Then Eq. (2.22) can be written as

$$
L_{\mu v} u_{v}=s_{\mu},
$$

where

$$
\left\{u_{v}\right\} \equiv\left\{m_{i}, \frac{p_{e}^{\prime}}{\gamma-1}, \rho^{\prime}\right\}
$$

is the five dimensional dependent variable vector, the five dimensional source vector $s_{\mu}$ is given by 


$$
s_{\mu} \equiv \frac{\partial}{\partial x_{j}}\left(e_{\mu j}^{\prime}-\widetilde{e}_{\mu j}\right)+\delta_{\mu 4}\left(e_{i j}^{\prime}-\widetilde{e}_{i j}\right) \frac{\partial \widetilde{v}_{i}}{\partial x_{j}} \text { for } \mu=1, \ldots 4
$$

and $\varepsilon_{4 j}^{\prime}=\eta_{j}^{\prime}$ and $\widetilde{\varepsilon}_{4 j}=\widetilde{\eta}_{j}$. This system can be formally solved by using the vector Greens function (Morse and Feshbach 1953, pp. 878-886) $g_{v \sigma}\left(x, t \mid x^{\prime}, t^{\prime}\right)$, which satisfies

$$
L_{\mu v} g_{v \sigma}=\delta_{\mu \sigma} \delta\left(x-x^{\prime}\right) \delta\left(t-t^{\prime}\right)
$$

to obtain

$$
u_{v}(\boldsymbol{x}, t)=\int_{-\infty}^{\infty} \int_{V} g_{v \mu}\left(\boldsymbol{x}, t \mid \boldsymbol{x}^{\prime}, t^{\prime}\right) s_{\mu}\left(\boldsymbol{x}^{\prime}, t^{\prime}\right) d \boldsymbol{x}^{\prime} d t^{\prime}
$$

and the derivatives can be transferred to the Greens function to obtain

$$
u_{v}(\boldsymbol{x}, t)=-\int_{-\infty}^{\infty} \int_{V} \gamma_{v j, \mu}\left(e_{\mu j}^{\prime}-\widetilde{e}_{\mu j}\right) d \boldsymbol{x}^{\prime} d t^{\prime}
$$

where

$$
\gamma_{v j, \mu}\left(x, t \mid x^{\prime}, t^{\prime}\right) \equiv \frac{\partial}{\partial x_{j}^{\prime}} g_{v \mu}+\frac{\partial \tilde{v}_{\mu}}{\partial x_{j}^{\prime}} g_{v 4}
$$




\section{References}

Bailly, C., Lafon, P., and Candel, S. (1995) A stochastic approach to compute noise generation and radiation of free turbulent flows, AIAA Paper No. 95-092.

Balsa, T.F., Gliebe, P.R., Kantola, R.A., Mani, R., Strings, E.J., and Wong, J.C.F., II (1978) High velocity jet noise source location and reduction, FAA Report No. FAA-RD-76-79.

Bastin, F., Lafon, P., and Candel, S. (1997) Computation of Jet Mixing Noise due to Coherent Structures: The Plane Jet Case, J. Fluid Mech., vol. 335, pp. 261-304.

Bechara, W., Bailly, C., Lafon, P., Candel, S. (1994) Stochastic approach to noise modeling, AIAA Journal, vol.no3.

Bers, A. 1975 Linear waves and instabilities. In Plasma Physics (ed. C.DeWitt \& J. Perraud ),pp. 113-2166 Gordon \& Beach.

Betchov, R. and Criminale, W.O. (1967) Stability of parallel flows, Academic Press.

Bodony, D.J. and Lele, S.K. (2002) Spatial scale decomposition of shear layer turbulence and the sound sources associated with the missing scales in a large-eddy simulation, AIAA Paper No. 2002-2454, $8^{\text {th }}$ AIAA/CEAS Aeroacoustics Conference, Breckenridge, $\mathrm{CO}$.

Bogey, C., Bailly, C., and Juvé, D. (2002) Computations of flow noise using source terms in linearized Euler equations, AIAA Journal, vol. 40, no. 2, pp. 235-243.

Bradshaw, P. (1978) Turbulence, Springer-Verlag, p. 6.

Briggs, R. J. 1964 Electron Stream Interaction with Plasmas. Massachusetts Institute of Technology Press.

Crighton, D.G. (1993) Computational aeroacoustics for low mach number flows, Computational Aeroacoustics, J.C. Hardin and M.Y. Hussani, eds., Springer-Verlag, NY.

Colonius, T., Lele, S.K. and Moin, P.(1997) Sound generation in a mixing layer. J. Fluid Mech., vol. 330, pp. 375-409.

Crow, S.C. (1970) Aerodynamic sound emission as a singular perturbation problem, Studies in Applied Math, vol. XLIX, no. 1, pp. 21-44.

Crow, S.C. and Champagne, F.H. (1971) Orderly structure in jet turbulence, J. Fluid Mech., vol. 48, pt. 3, pp. 547-592.

Dowling, A.P., Ffowcs Williams, J.E., and Goldstein, M.E. (1978) Sound propagation in a moving stream, Phil. Trans. Roy. Soc. Lond., A, vol. 288, no. 1353, pp. 321-349. 
Freund, J.B. (2001) Noise sources in a low-Reynolds-number turbulent jet at Mach 0.9, J. Fluid Mech., vol. 438, pp. 277-305.

Freund, J.B. and Fleischman, T.G. (2002) Ray traces through unsteady jet turbulence, International Journal of Aeroacoustics, vol. 1, no. 1, pp. 83-96.

Hardin, J.C. and Pope, D.S. (1994) An acoustic splitting technique for computational aeroacoustics, Theoretical and Computational Fluid Dynamics, vol. 6, no. 5-6, pp. 334-340.

Gaster, M., Kit, E., and Wygananski, I.(1985) Large scale structures in a forced turbulent mixing layer, J. Fluid Mech., 150, pp. 23-39.

Goldstein, M.E. (1976) Aeroacoustics, McGraw-Hill.

Goldstein, M.E. (1999) Some recent developments in jet noise modeling, Program and Abstracts of the $6^{\text {th }}$ International Congress on Sound and Vibration, Copenhagen, Denmark, p. 21.

Goldstein, M.E. (2000) Some recent developments in jet noise modeling, Program of the $38^{\text {th }}$ AIAA Aerospace Sciences Meeting and Exhibit, Reno, Nevada.

Goldstein, M.E. (2002) A unified approach to some recent developments in jet noise theory, International Journal of Aeroacoustics, vol. 1, no. 1, pp. 1-16.

Hussain, A.M.K.F. (1983) Coherent structures-reality and myth. Phys Fluids, 26 pp. 2816-2850.

Lele, S.K. (1994) Compressibility effects in turbulence, Annual Rev. of Fluid Mech., vol. 26, pp. 211-254.

Lighthill, M.J. (1952) On sound generated aerodynamically: I. General theory, Proc. R. Soc. Lond., A211, pp. 564-587.

Lighthill, M.J. (1954) On sound generated aerodynamically: II. Turbulence as a source of sound, Proc. R. Soc. Lond. A222, pp. 1-32.

Lighthill, M.J. (1963) 'Jet noise,' The Wright Brothers Lecture of 1963, AIAA Journal, vol. 1, pp. 1507-1517.

Lilley, G.M. (1974) On the noise from jets, Noise Mechanism, AGARD-CP-131, pp. 13.1-13.12.

Lilley, G.M. (1996) The radiated noise from isotropic turbulence with applications to the theory of jet noise, J. Sound and Vib., vol. 190, no. 3, pp. 463-476.

Liu, J.T.C.(1988)Contributions to the understanding of large scale coherent structures in developing free shear flows, .Advances in Applied Mech., vol. 26, pp. 183-309. 
Mankbadi, R. and Liu, J.T.C. (1984) Sound generated aerodynamically revisited: large scale structures in a turbulent jet as a source of sound, Phil. Trans., Roy. Soc. Lond., A, 311, pp. 183-217.

Morkovin, M.V. (1962) Effects of compressibility on turbulent flows, Mechanique de la Turbulence, A. Favre, ed., CNRS, pp. 367-380, Paris.

Monin, A. and Yaglom, A. (1971) Statistical hydrodynamics (English translation), MIT Press, p. 206.

Morse, P.M. and Feshbach, H. (1953) Methods of theoretical physics, McGraw-Hill.

Millioschikov, M.D.(1941) Theory of homogeneous isotropic turbulence, Doklady Akademii Nauk SSSR, vol. 32, no. 9, pp. 611-614.

Phillips, O.M. (1960) On the generation of sound by supersonic turbulent shear layers, J. Fluid Mech., vol. 9, pp. 1-28.

Pridmore-Brown, D.C. (1958) Sound propagation in a fluid flowing through an attenuating duct, J. Fluid Mech., vol. 4, pp. 393-406.

Reau, N. and Tumin, A. (2002a) On harmonic perturbations in a turbulent mixing layer, European J. Mech. B/Fluids, 21, pp.143-155.

Reau, N. and Tumin, A. (2002b) Harmonic perturbations in turbulent wakes, AIAA J., 40, pp. 526-530.

Reynolds, W.C., and Hussain, A.K.M.F., (1972) The mechanics of an organized wave in turbulent shear flow,. 3, Theoretical models and comparison with experiments. J. Fluid Mech. 54, pp. 263-288.

Ribner, H.S. (1962) Aerodynamic sound from fluid dilatations, University of Toronto Inst. Aerophysics Report no. 86 .

Roe, P.L. (1992) AIAA Paper No. 92-02-032, DGLR/AIAA Aeroacoustics Conference.

Rogallo, R.S. and Moin, P. (1884) Numerical simulation of turbulent flows, Ann. Rev. Fluid Mech., vol. 16, pp. 99-137.

Smits, A.J. and Dussauge (1996) Turbulent shear layers in supersonic flow, AIP Press, New York.

Speziale, C.G. (1991) Analytical methods for the development of Reynolds-stress closure in turbulence, Ann. Rev. Fluid Mech., vol. 23, pp. 107-157.

Speziale, C.G. and So, M.C. (1998) Turbulence modeling an simulation in the Handbook of Fluid Dynamics, Richard Johnson, ed., CRC Press.

Tam, C.K.W. (1998) Jet noise: since 1952, Theoretical and Computational Fluid Dynamics, vol. 10, pp. 393-405. 
Tam, C.K.W. and Morris, P.J.(1980) The radiation of sound by instability waves of a compressible plane turbulent mixing layer, J. of Fluid Mech., 98, pp. 349-381.

Tam, C.K.W. and Auriault, L. (1999) Jet mixing noise from fine-scale turbulence, AIAA Journal, vol. 37, no. 2, pp. $145-153$.

Tam, C.K.W., Golebiowski, M., and Seiner, J.M. (1996) On the two components of turbulent mixing noise from supersonic jets, AIAA Paper No. 96-1716.

Zhao, W. Frankel, S.H., and Mongeau, L. (2000) Effects of spatial filtering on sound radiation from a subsonic axisymmetric jet, AIAA Journal, vol. 38, no. 11, pp. 2032-2039. 